\title{
SUPRACONDYLAR-Y FRACTURES OF THE HUMERUS
}

\author{
E. Mervyn Evans, Swansea, Wales
}

The supracondylar-Y fracture of the humerus is one of those uncommon injuries whose treatment varies considerably from one clinic to another. It is caused by a heavy fall on the hand or point of the elbow, the olecranon being forced upwards as a wedge between the humeral condyles, prizing them apart and displacing them upwards and backwards. Displacement is considerable, and the fracture is unstable; the violence of the injury sometimes causes an associated vascular or nerve lesion. The fracture occurs usually in adolescent or in adult life.

It is a difficult fracture to treat, and the very diversity of views on the subject is an indication of the poor quality of the results often obtained. In the words of Watson-Jones (1943) " no injury of the elbow gives rise to more difficulty in treatment. It may be impossible to secure perfect replacement by manipulation and traction. Operative reduction is very difficult; it involves a free dissection of the triceps and capsule of the joint, and there is usually no natural stability of the fragments, so that reduction can only be maintained by screws and plates. Adhesion of the triceps, contraction of the capsule, avascular necrosis contribute to dense adhesion formation and permanent stiffness. Despite the perfect anatomical reposition which may have been secured the final range of movement is usually less satisfactory than after manipulation, even when manipulative reduction has been less accurate. Operative reduction is not justified by the results. Two alternatives are available: 1) To secure the best possible position by manipulation and traction. 2) In grossly comminuted fractures with irreparable joint injury to perform an immediate arthroplasty." Watson-Jones goes on to describe manipulative reduction followed by plaster immobilisation, a method which the author has found to be followed by considerable joint stiffness. Perhaps more commonly used is the so-called "bag of bones" technique, whereby the arm is supported in a collar and cuff, and immediate active movements practised. This is certainly a valuable method in the elderly, as a considerable range of movement may be obtained. The joint, however, is apt to be unstable and much power is lost, so that it cannot be said to lead to a satisfactory result in the younger patient. Late traumatic arthritis must not be forgotten; the elbow is a non-weight-bearing joint, but gross disorganisation of the joint surfaces does lead to arthritis in time.

If operative reduction is advocated the Campbell posterior approach is most favoured, with accurate fixation of all the main fragments by plates or multiple screws-a procedure calculated to lead to considerable limitation of movement. Surely a simpler approach to the treatment of this difficult injury is needed. The ordinary supracondylar fracture of the humerus is not difficult, and the $\mathrm{Y}$-fracture can be converted into a supracondylar by the accurate reduction and fixation of the condylar fragments. Furthermore, the alignment of the intraarticular surfaces is thus restored, and good lasting function may be expected, provided the operative approach has done no harm. Any residual displacement between the two fixed condylar fragments and the shaft may be corrected by subsequent manipulation, and, even if it persists, will not have any great deleterious effect upon the ultimate function.

\section{TEGHNIQUE OF OPERATION}

The key to the success of this simplified method of internal fixation lies in the exposure, and here the injury itself makes things easier for us. Provided the operation is undertaken within a reasonably short time of the accident, a vertical incision on either side of the elbow joint will lead one straight into the fracture haematoma. The flexor and extensor muscles 
have been stripped from the surfaces of the humerus by the injury, and little further stripping is necessary; they can be retracted easily without further damage, and are thus preserved for early function. The joint as a rule is found widely open; if not, it is distended with blood, and an incision through the capsule gives free exposure. The approach is made from the side of the smaller condylar fragment; if this is on the medial side, as is the more usual, the ulnar
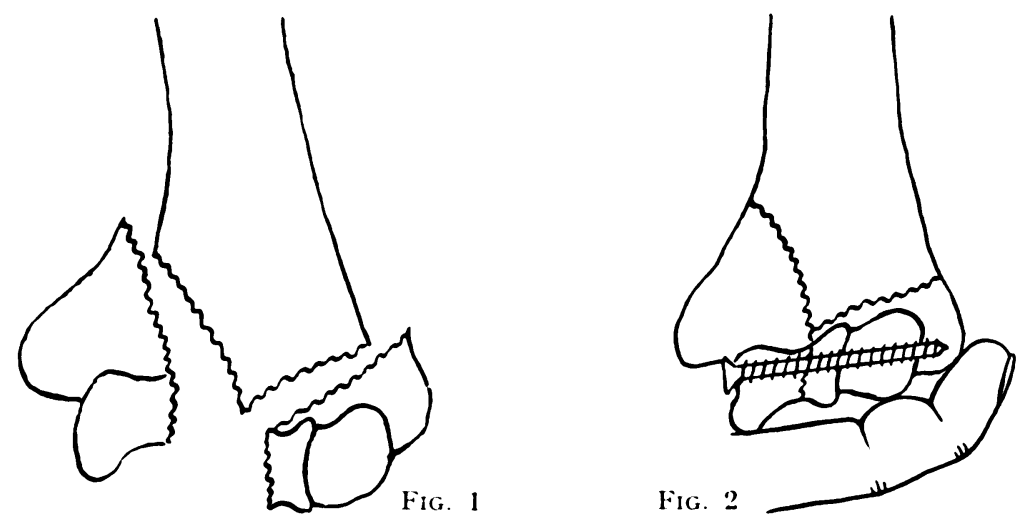

Fixation of the condylar fragments of a $\mathrm{Y}$-shaped supracondylar fracture with a screw.

Thereafter the management is that of an uncomplicated supracondylar fracture.

nerve should be exposed early in the dissection, and may be transposed anteriorly if its course lies near the fracture site. When the fracture has been exposed, the index finger in the joint hooks the far condyle back into position against the near one (Figs. I and 2 ). The break between the two is sharp and clear cut, and it is not difficult to secure apposition by feel alone. While the finger holds the position a seven sixty-fourths inch drill is inserted through the cancellous core of the two condyles, and its position checked radiographically. If the position is satisfactory, the drill is replaced by a long screw, the finger being kept in position during the whole procedure (Fig. - $)$ ). When accurate apposition cannot be attained by manipulation, a seven sixty-fourths inch drill hole is made in the distal condylar fragment, and a nine sixty-fourths inch hole in the proximal; a screw is then passed through the proximal into the distal fragment, and the two approximated. The united condylar fragments are then manipulated, under direct vision, into as good a position as possible with the shaft, and the wound closed. External fixation is by

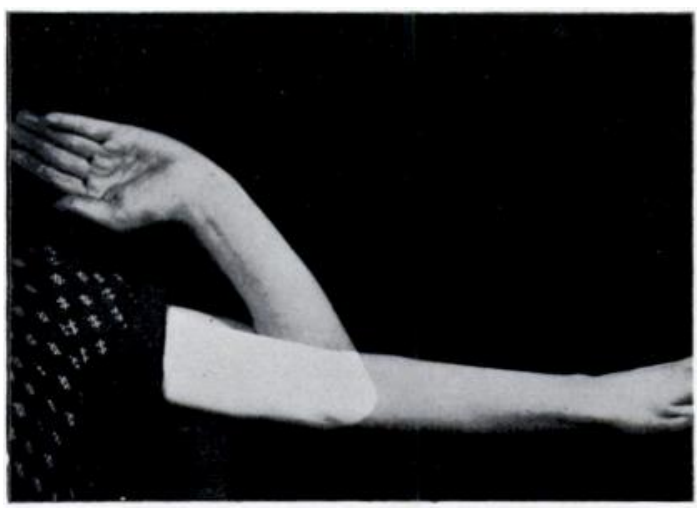

FIG. 3

Case 1 - Showing full movement a vear after operation. pressure bandage alone, in moderate flexion, for three weeks, after which active movements are started as soon as the stability of the supracondylar element of the fracture allows.

The operation has been performed in six cases, five of which are reported in detail; the sixth was in a woman of sixty-five whose elbow stiffened remarkably quickly, for after three weeks she had virtually no movement; it would perhaps be better if operative reduction were reserved for adolescents and young adults, and the "bag of bones" technique used in the older patients.

\section{CASE REPORTS}

Case 1-A girl aged fifteen sustained a closed comminuted fracture of the lower end of the left humerus and a fracture of the lower end of the left radius as the result of a fall on to the hand 
at gym. The elbow fracture was approached from the medial side, and a temporary screw was used to hold the upwardly displaced medial humeral fragment while the condyles were being fixed; it was then removed. Active movements were started at six weeks; the final range of elbow movement was full (Fig. 3), and the power normal.

Case 2-A woman aged thirty-five sustained a closed supracondylar- $Y$ fracture of the right humerus by falling from a horse (Fig. 4). There was extensive bruising around the joint, and a complete median nerve palsy on admission. At operation through a lateral approach, five hours after the accident, both the drill and the screw were placed rather high, but an adequate hold on both condylar fragments was obtained (Fig. 5). Active movements were begun five weeks later, and four months after the operation the range of elbow movement was from 160 to 60 degrees, with good muscle power. The median paralysis persisted; the nerve was eventually explored, and found embedded in scar tissue. After it had been freed, full recovery was obtained (Fig. 6).

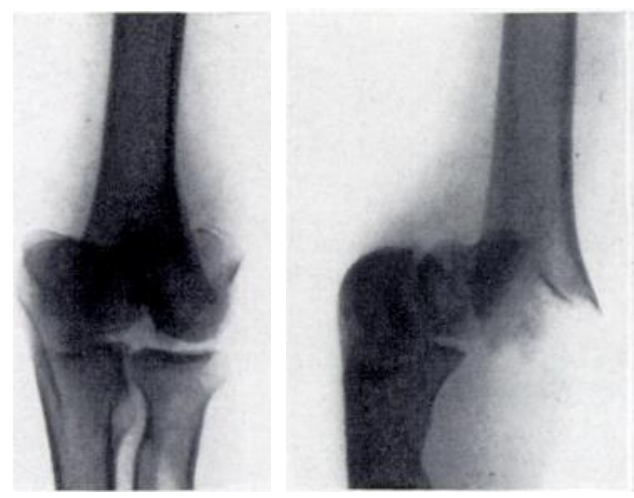

Fic. 4

Case 2 - Before treatment

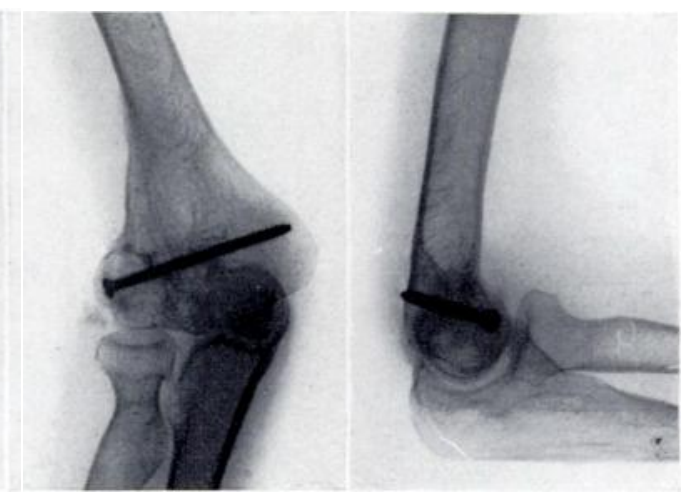

FIG. 5

Case 2-Fifteen months after operation.

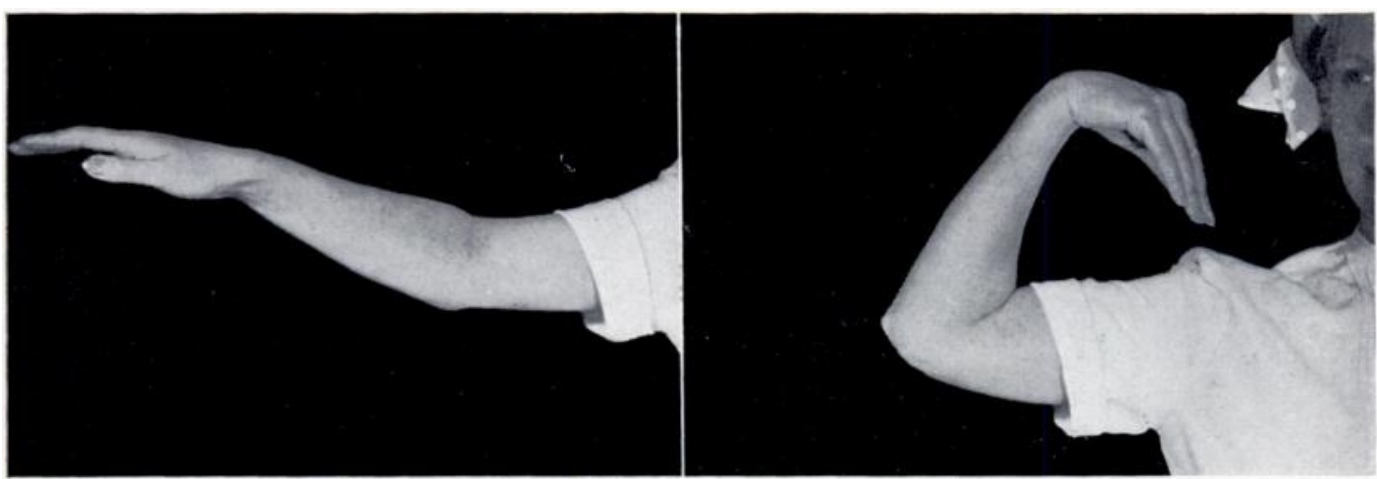

FIG. 6

Case 2-The range of elbow movement fifteen months after operation.

Case 3 A boy aged fifteen was admitted three days after a motor accident, having been treated in the first place in another hospital. He had sustained a comminuted compound fracture of the lower end of the left humerus, with a three-inch transverse wound posteriorly involving the triceps; the wound had been cleaned, packed with vaseline gauze and left open. It was felt that a stiff, possibly infected elbow joint was likely if it were left alone, and it was decided to attempt open reduction. Six days after the accident the posterior wound was sutured, and the condylar fragments fixed by a transverse screw, placed from the medial side. Active movements were begun four weeks later, and the fracture united with good joint alignment; there was, however, some forward displacement at the supracondylar site. His final range was from 145 to $\mathbf{5 0}$ degrees, which may be regarded as satisfactory, in view of the severity of his original injury. The power was normal, and he was free from pain.

vol. 35 B, No. 3 , August 1953 
Case 4 - A girl aged eleven sustained a compound supracondylar- $Y$ fracture of the right humerus, there being a small puncture wound at the back of the joint. In this case the fall was on to the hand, as is suggested by the upward displacement of the large fragment on the ulnar side (Fig. 7). This appears to be a comparatively common fracture pattern, and strong longitudinal traction on the limb is needed to obtain reduction. The condylar fragments were fixed with a screw (Fig. 8). Active movements were started six weeks after operation, and less than three months from the date of the accident she had an elbow range of from 170 to 60 degrees (Fig. 9). At this stage she left the country; she was still improving, and it may be supposed that her final range was greater.

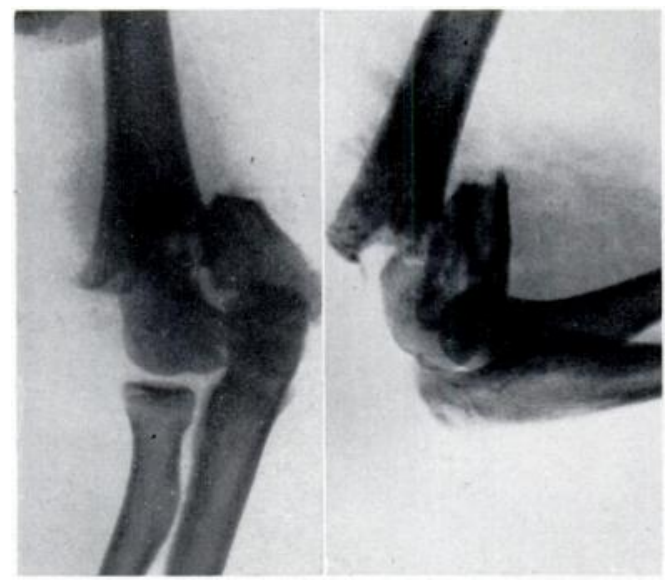

FIG. 7

Case 4-Before treatment.

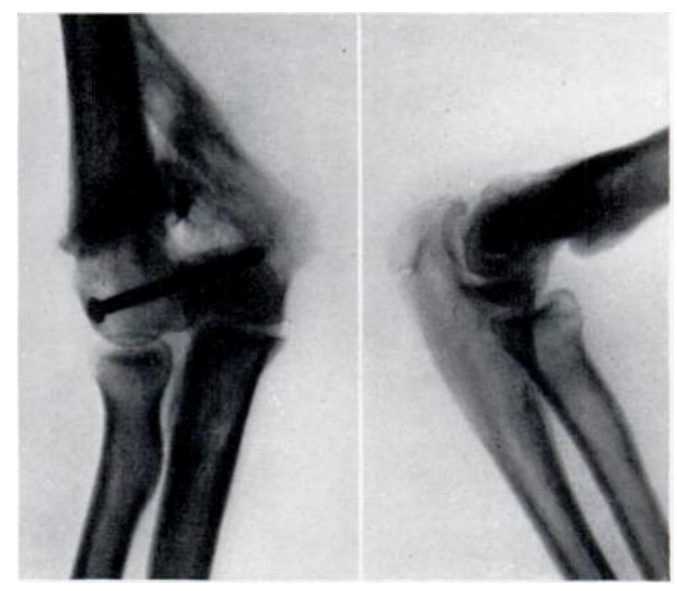

FIG. 8

Case 4-Ten weeks after operation.

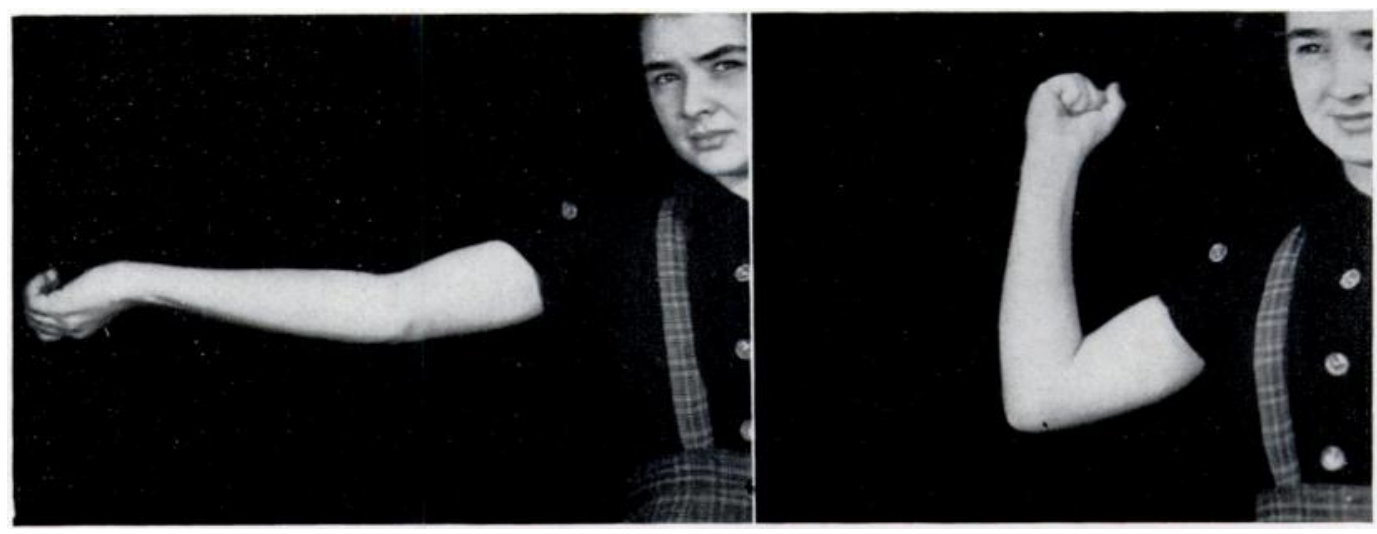

FIG. 9

Case 4-Range of movement ten weeks after operation.

Case 5-A man aged forty-one fell from a ladder on to his outstretched left arm, sustaining a closed supracondylar- $Y$ fracture of the left humerus and a fracture of the lower end of the radius; the upward displacement of the ulnar condylar fragment is clearly seen (Fig. 10). The condyles were fixed by a transverse screw placed from the medial side (Fig. 11); the ulnar nerve was found crossing the fracture site at the time of the operation, and was transposed anteriorly. Active movements were started at three and a half weeks, and the final range of movement was from 80 degrees to full extension (Fig. 12); the power was normal, and he had returned to his preaccident work as an electro-plater.

\section{DISCUSSION}

It must be admitted that five cases provide slender evidence on which to base a claim for a new method of treatment. The supracondylar-Y fracture of the humerus is uncommon, 
however, and a large series would be difficult to obtain. The accepted methods of treatment are not satisfactory. Manipulation is uncertain, and requires plaster fixation for retention of position, so that the final function is often poor. The "bag of bones " technique is useful in the elderly, but gives a weak unstable elbow, not acceptable to the young or to the manual worker. Accurate reconstruction is perhaps the least satisfactory method of all, for it entails wide exposure, with consequent damage to important muscle groups. It is felt that screwfixation of the condylar fragments is reasonable, in that it takes care of the essential

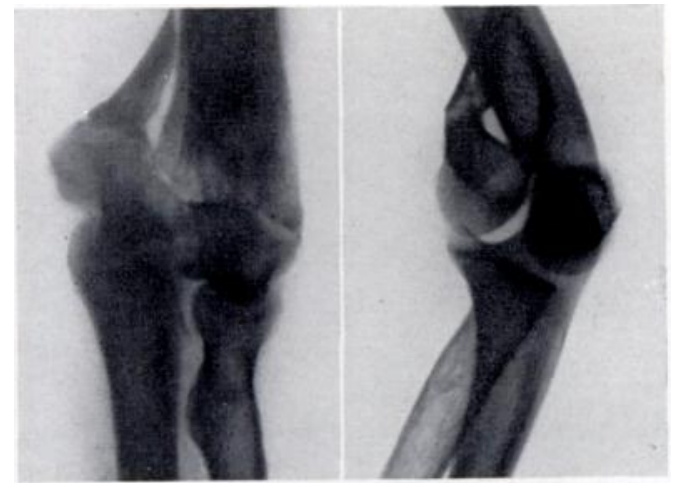

FIG. 10

Case 5.-Before treatment.

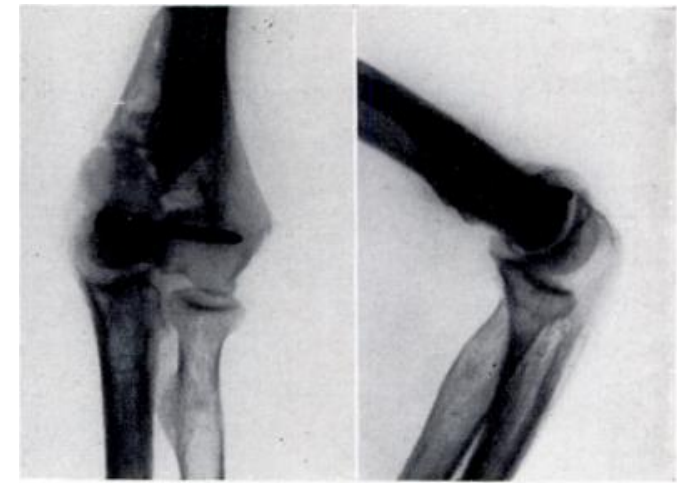

FIG. 11

Case 5-Eight months after operation.

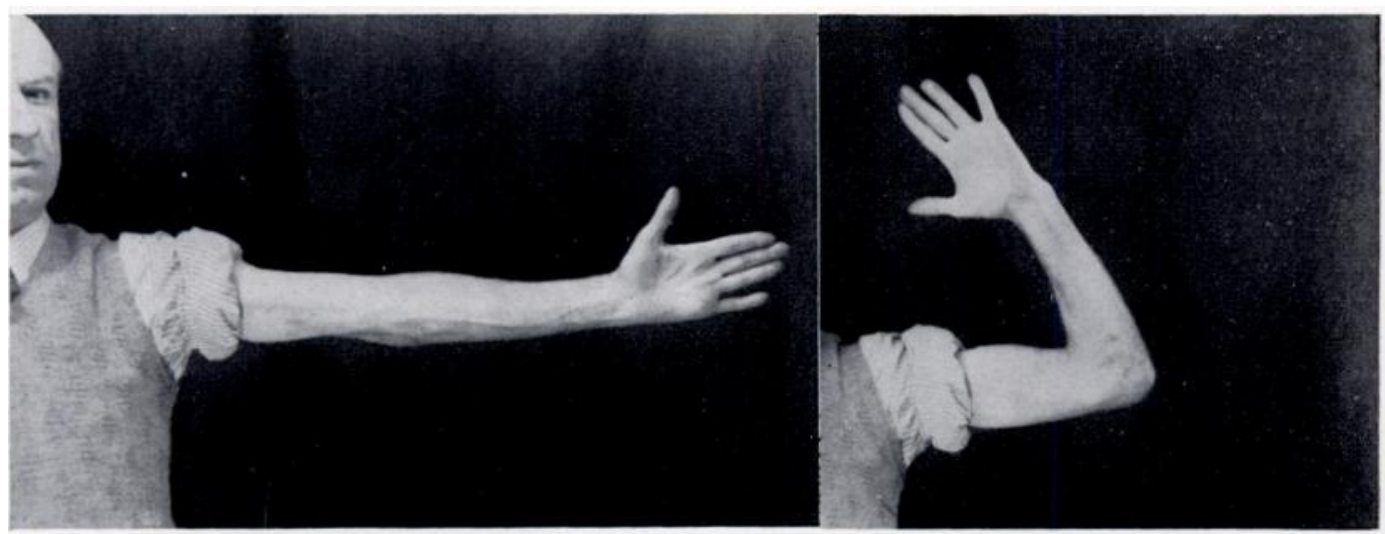

FIG. 12

Case 5-Range of movement eight months after operation.

displacement and restores joint alignment, while the supracondylar element is dealt with by manipulation. The operation can be performed without damage to the important flexor and extensor muscle groups, and relatively early movements may be instituted without fear of redisplacement. One cannot expect a perfect result in this fracture, but by condylar fixation one should be able to rely on 60 degrees of movement, complete stability, and adequate muscle power.

I am indebted to Mr Gill of the Photographic Department of the Birmingham Accident Hospital for the preparation of the illustrations.

\section{REFERENCE}

Watson-Jones, R. (1943) : Fractures and Joint Injuries. Third edition. Edinburgh: E. \& S. Livingstone Ltd. vol. 35 B, No. 3, AUgust 1953 